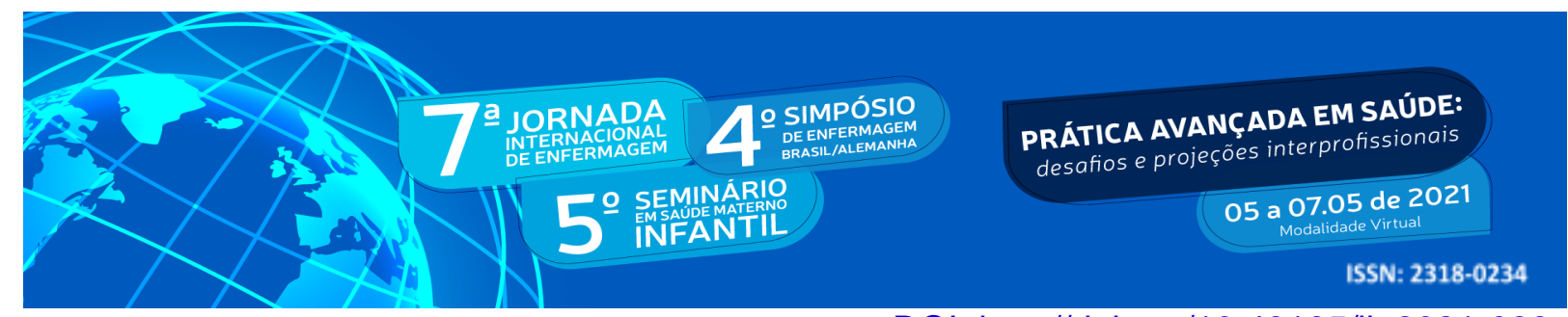

\title{
A PERCEPÇÃO DOS PROFISSIONAIS DA SAÚDE PARA POLÍTICAS PÚBLICAS DE INCLUSÃO NO CONTEXTO DA VULNERABILIDADE ${ }^{1}$
}

\author{
Carolina Araujo Londero ${ }^{2}$; Stephani Sortica Fantinel ${ }^{3}$; Munah Najeh Saleh Ahmad \\ Maruf $^{4}$; Bruna Weis ${ }^{5}$; Chanelle Moraes ${ }^{6}$; Martha Helena Teixeira de Souza ${ }^{7}$
}

\begin{abstract}
RESUMO
A partir do cenário atual, implica-se refletir a percepção dos profissionais da saúde no que tange às políticas públicas, sendo a atenção primária em saúde como foco do estudo, por se configurar como a porta de entrada dos serviços de saúde. O objetivo foi compreender como os profissionais da saúde da atenção básica percebem o contexto da vulnerabilidade social nos serviços públicos de saúde. Apresenta-se uma pesquisa descritiva e exploratória de caráter qualitativo, sendo dados analisados segundo os direcionamentos de Minayo (2010). Aprovado pelo comitê de ética em pesquisa (CEP), sendo este 4.350.543. A coleta de dados foi realizada em Unidades Básicas de Saúde, mediante a técnica de entrevistas com questões norteadoras, no período de outubro de 2020. Com a análise de dados compreendeu-se que as políticas públicas no contexto de vulnerabilidade existem e, ademais deve-se articular a gestão pública, para que as demandas dessa população sejam contempladas.
\end{abstract}

Palavras-chave: Vulnerabilidade; Assistência; Profissionais.

\begin{abstract}
From the current scenario, it is necessary to reflect on the perception of health professionals regarding public policies, with primary health care as the focus of the study, because it is configured as the gateway to health services. The objective was to understand how primary care health professionals perceive the context of social vulnerability in public health services. This is a descriptive and exploratory qualitative research, with data analyzed according to the guidelines of Minayo (2010). Approved by the Research Ethics Committee (CEP), being this 4.350.543. Data collection was carried out in Unidades Básicas de Saúde (Basic Health Units), through the technique of interviews with guiding questions, in the period of October 2020. With the data analysis, it was understood that public policies exist in the context of vulnerability and, furthermore, public management must be articulated so that the demands of this population are contemplated.
\end{abstract}

Key Words: Vulnerability; Assistance; Professionals.

\footnotetext{
${ }^{1}$ A percepção dos profissionais da saúde para políticas públicas de inclusão no contexto da vulnerabilidade. Trabalho completo, possuindo amparo Fapergs.

${ }^{2}$ Estudante do Curso de Enfermagem. Universidade Franciscana. E-mail: carolina.alondero@gmail.com

${ }^{3}$ Estudante do Curso de Enfermagem. Universidade Franciscana. E-mail: stephanifantinel@gmail.com

${ }^{4}$ Estudante do Curso de Enfermagem. Universidade Franciscana. E-mail: munah saleh@hotmail.com

${ }^{5}$ Estudante do Curso de Medicina. Universidade Franciscana. E-mail: Bruna weis@hotmail.com

${ }^{6}$ Mestranda Materno infantil. Universidade Franciscana. E-mail: chanellemoraes@hotmail.com

${ }^{7}$ Professora orientadora: Doutora em Ciências. Universidade Franciscana. E- mail: marthahts@gmail.com
} 


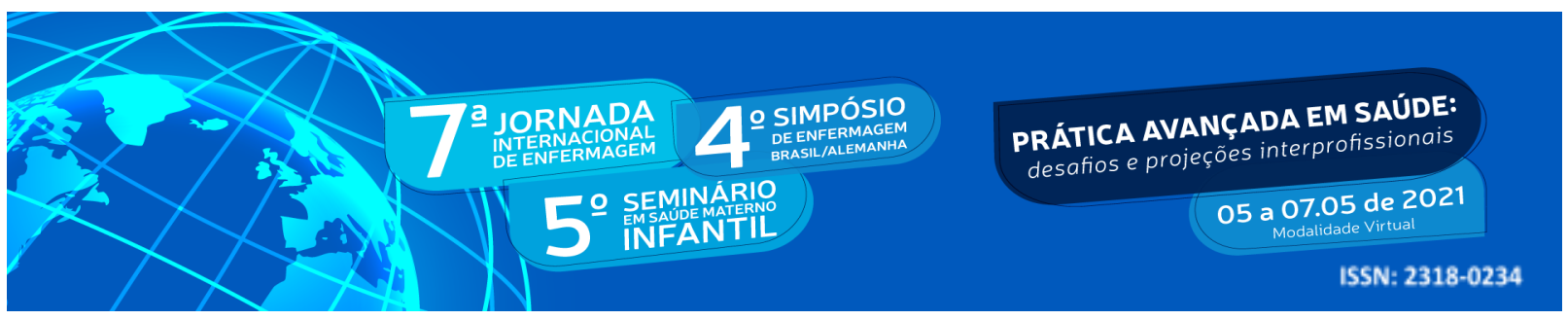

\section{INTRODUÇÃO}

$\mathrm{Na}$ literatura científica o termo vulnerabilidade possui conceitos diferentes, a partir da década de 80 se intensificaram os estudos que tratam da vulnerabilidade como quadro conceitual. A vulnerabilidade busca expressar os potenciais de adoecimento ou de não adoecimento relacionados a todo e cada indivíduo que vive em um conjunto social (NICHIATA et al, 2008).

O termo vulnerabilidade na área da saúde começou a ser perpetuado no ano de 1980, durante os estudos sobre a síndrome da imunodeficiência adquirida (Aids), sendo empregado para designar suscetibilidades das pessoas a problemas e danos de saúde. Podendo ser relacionada às populações pobres ou menos favorecidas economicamente, porém isto não é um fator determinante, a temática vulnerabilidade está condicionada à ausência ou precariedade no acesso à renda, mas atrelada também às fragilidades de vínculos afetivo-relacionais e desigualdade de acesso a bens e serviços públicos (CARMO et al, 2018).

No Brasil as diferentes condições sociais, econômicas, políticas e culturais dos sujeitos contribui para a percepção de como este irá buscar a assistência, desta forma há uma necessidade para construção de planos de cuidado voltados ao indivíduo. Sendo assim, faz-se necessário o profissional conhecer as políticas públicas voltadas a populações vulneráveis, com isso o usuário terá autônoma (BARRA et al, 2010).

As necessidades de saúde muito circulam como a questão da Atenção Primária, em estudos sobre o acesso a serviços e cuidados, a qualidade da assistência e das práticas dos profissionais, e ainda sobre os direitos à saúde e deveres do Estado em suas políticas públicas saúde (SCHRAIBER, 2012). Vale ressaltar que foi durante a reforma sanitária e estruturação da Saúde Coletiva, vinculando-se à democratização do estado brasileiro e à reforma da saúde que começou a ser questionado as condições socioeconômicas como determinantes de saúde.

A estruturação do Sistema Único de Saúde (SUS), traz o usuário como ativo no seu processo de cuidado, sendo assim os indivíduos têm um grande papel no processo de reivindicação dos seus direitos fundamentais (SILVA et al, 2012). Destaca-se que a vulnerabilidade social influencia diretamente no contexto de saúde brasileira. 


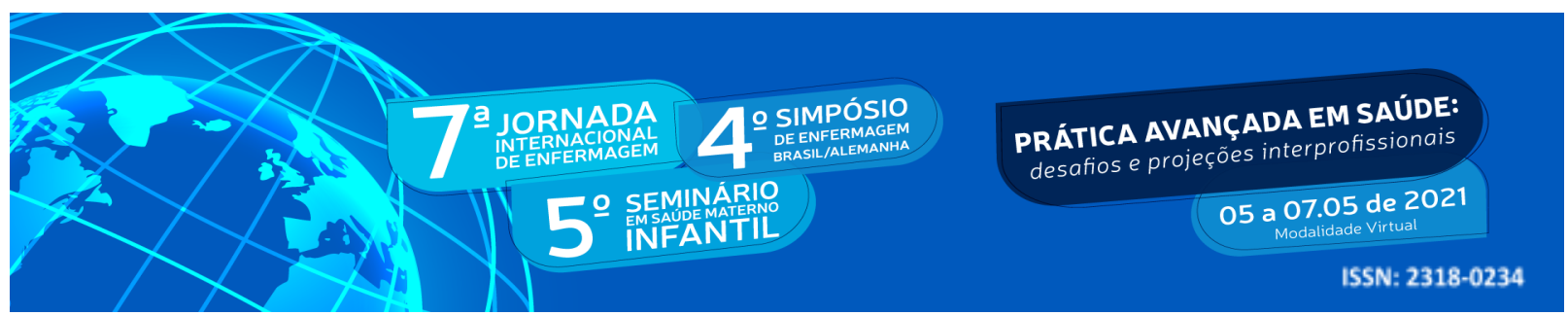

Um dos grandes desafios do SUS é produzir saúde de uma forma que o usuário seja o protagonista do seu cuidado, no contexto de atendimento de populações vulneráveis, deve-se articular estratégias para que estes tenham entendimento do processo saúde-doença (VASCONCELOS, FELIX, GATTO, 2017). A estimulação deste protagonismo pode ocorrer nos serviços de saúde, a partir da capacitação dos profissionais envolvidos, dentre eles, os gestores, com isso o fortalecimento do debate acerca das questões de vulnerabilidade social, atreladas a gestão pública junto aos serviços de saúde.

A partir do cenário atual, implica-se refletir a percepção dos profissionais da saúde no que tange às políticas públicas, sendo a atenção primária em saúde como foco do estudo, por se configurar como a porta de entrada dos serviços de saúde. Segundo o artigo nove do decreto $\mathrm{N}^{\mathrm{o}} 7.508$, os serviços de saúde nas Redes de Atenção à Saúde, como as Unidades Básicas de Saúde e Estratégias de Saúde da Família são configuradas como portas de entrada à assistência à saúde (BRASIL, 2011).

Os princípios do SUS representam o reconhecimento da complexidade e das necessidades de cada indivíduo, demonstrando que o direito à saúde no Brasil não está restrito apenas para uma parte da população, mas deve contemplar o essencial para um cuidado à saúde com dignidade (SILVA et al, 2012). Assim, faz-se necessário compreender os contextos sociais e de vulnerabilidade da sociedade brasileira.

Pensar no papel do profissional da saúde para a temática, é importante, pois os profissionais estão diariamente inseridos em diversos contextos de saúde. Assim, a relevância do conhecimento sobre a vulnerabilidade está nas implicações que produz na saúde daqueles que estão em situação de vulnerabilidade e, consequentemente, na identificação das suas necessidades de saúde (BERTOLOZZI et al, 2009).

\section{OBJETIVO}

Compreender como os profissionais da saúde da atenção básica percebem o contexto da vulnerabilidade social nos serviços públicos de saúde.

\section{METODOLOGIA}




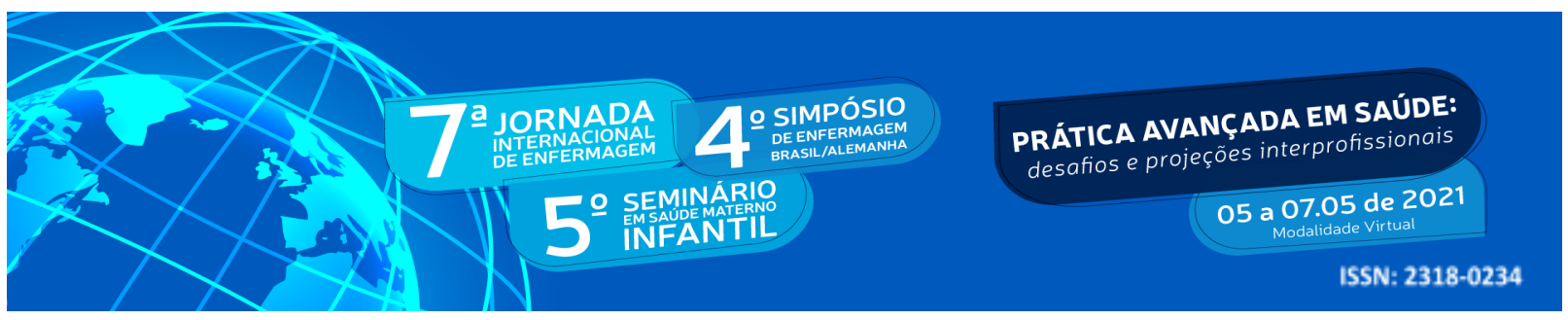

O presente trabalho é uma pesquisa descritiva exploratória de caráter qualitativo e possui fomento da bolsa de pesquisa da Fundação de Amparo à Pesquisa do Estado do Rio grande do Sul (Fapergs), o qual foi aprovado pelo Comitê de Ética em Pesquisa da Universidade Franciscana (CEP), sendo este 4.350.543.

Compunham sujeitos da pesquisa, profissionais de saúde que trabalham no Sistema Único de Saúde da região central do Rio Grande do Sul. Para critérios de inclusão foi: Atuar no serviço público de saúde por mais de seis meses junto a populações vulneráveis e assinar voluntariamente o Termo de Consentimento Livre e Esclarecido (TCLE), sendo os critérios de exclusão: Aqueles que não quiseram participar ou não estavam atuando nos serviços de saúde no dia das entrevistas.

A coleta de dados foi realizada em Unidades Básicas de Saúde, mediante a técnica de entrevistas com questões norteadoras, ocorrendo assim a interação entre pesquisador e participantes, no período de outubro de 2020. Usou-se um questionário semiestruturado, com questões norteadoras, possibilitando a interação entre pesquisador e participante, construindo um diálogo com foco pré-estabelecido.

A análise dos dados ocorreram com as orientações metodológicas de (Minayo 2010) que preconizou-se os seguintes passos: A ordenação dos dados coletados, a classificação dos dados e a análise final, contando os objetivos da pesquisa e os temas que emergiram das entrevistas. Após a concretização deste processo, realizaremos uma discussão dos achados, a aproximação com a literatura já existente e pertinente à temática.

Para tanto, o referencial teórico utilizado apoiou-se nas bases de dados Literatura Latino-Americana e do Caribe em Ciências da Saúde (LILACS) e Scientific Eletronic Library Online (SCIELO).

\section{RESULTADOS E DISCUSSÃO}

A partir da Constituição de 1988, configura-se, no Brasil, um cenário no qual as políticas sociais entraram em ascensão, graças a um processo de ampliação dos direitos 


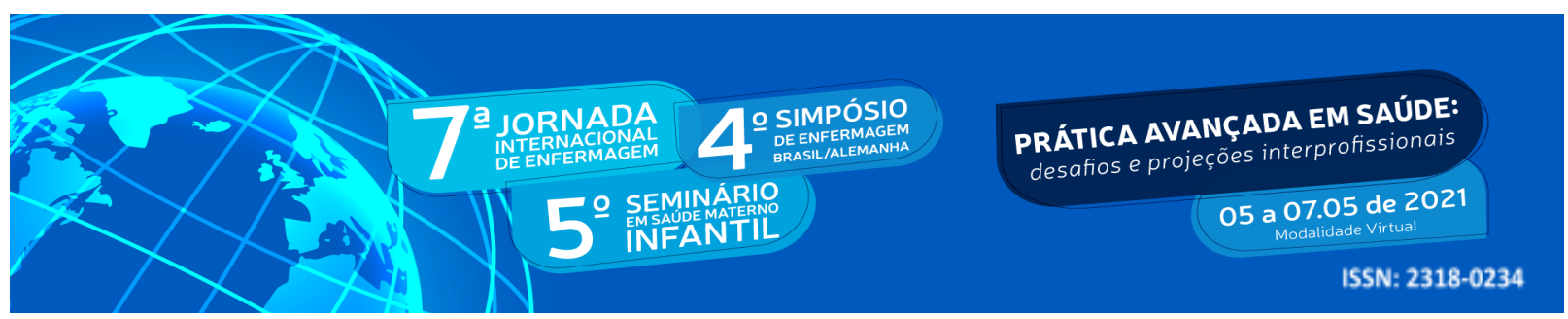

humanos e sociais. As políticas sociais visam responder às demandas advindas das relações contraditórias determinadas pelas lutas de classes, raça, gênero e etnia (MENDES et al, 2019).

No que se refere às esferas das políticas públicas de saúde, estas estão vinculadas aos serviços, a democratização do acesso e do reconhecimento dos direitos humanos e sociais, estes já estão presentes para a elaboração de programas de atenção específicos (SCHRAIBER, 2012). Para tanto cita-se a Política Nacional de Humanização (PNH) de 2013, que assegura a comunicação entre os trabalhadores e os usuários, para assim construir práticas que auxiliem na autonomia dos usuários.

A forma como o sujeito busca as Unidades Básicas de Saúde está diretamente ligado a como estes serviços acolhem, visto isso, o seguinte relato de uma profissional "Pra nós aqui a vulnerabilidade é bem grande, bem presente mesmo, a gente procura atender todos sem discriminação nenhuma”. A realização deste acolhimento pode assegurar que o usuário retorne ao serviço de saúde, sendo isto uma postura ética que implica na escuta do usuário em suas queixas, no reconhecimento do seu protagonismo no processo de saúde e adoecimento, e na responsabilização pela resolução, com ativação de redes de compartilhamento de saberes (BRASIL, 2013).

Desta forma foi questionado qual a percepção dos profissionais, no que tange às políticas públicas de saúde voltadas às populações vulneráveis, visando compreender a resolutividade destas no cenário que estes profissionais estão inseridos, sendo a fala de um deles "Elas atendem, mas não de forma efetiva". Para tanto a Lei 8080 ou também conhecida como Lei Orgânica do SUS, assegura saúde como um direito fundamental do ser humano, sendo o Estado provedor das condições indispensáveis ao seu pleno exercício (BRASIL, 1990).

Em seu artigo $3^{\circ}$ a lei Orgânica do SUS (1990) aborda que os níveis de saúde expressam a organização social e econômica do País, observando a saúde como um dos determinantes sociais, atrelado a isso alimentação, moradia e saneamento básico. Ademais foi relatado que "Existe bastante vulnerabilidade social aqui, as vezes as pessoas não tem nem água encanada”.

Segundo dados de 2019 do Instituto Trata Brasil, cerca de apenas 54,1\% da população brasileira tem acesso à coleta de esgoto, sendo isso quase 100 milhões de brasileiros sem 


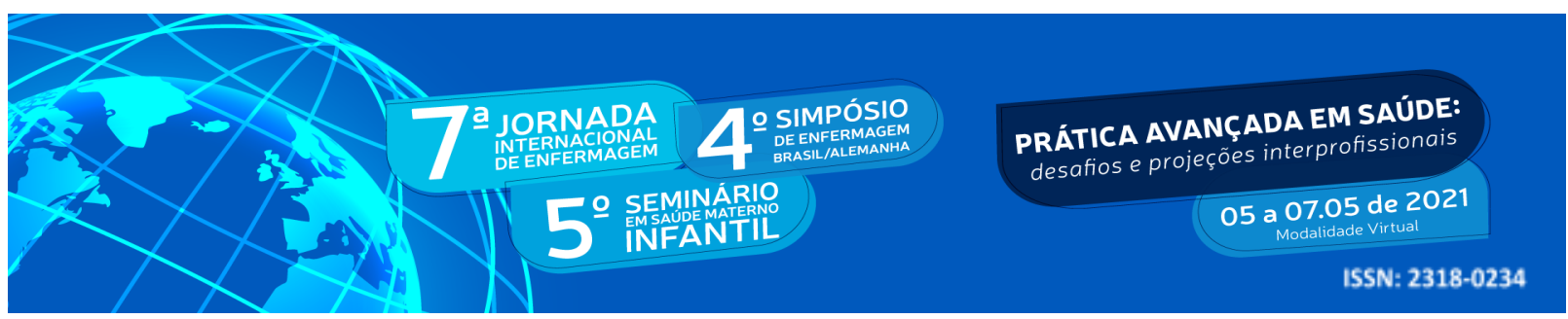

acesso a este serviço. A nível da região Sul 46,3\% da população possui acesso a coleta de esgoto (KLASSMANN; CARBONAI, 2020).

A Organização Mundial da Saúde afirma saúde como um bem-estar físico, mental e social, refletindo sobre esta falta de acesso a um dos serviços básicos para a higiene, afirma-se que há a necessidade de debater mais com o poder público no que tange às políticas públicas de saúde para as populações que vivem em vulnerabilidade social.

\section{CONCLUSÃO}

Com a análise de dados compreendeu-se que as políticas públicas no contexto de vulnerabilidade existem e, ademais deve-se articular a gestão pública, para que as demandas dessa população sejam contempladas. Tornando-se cada vez mais necessário a incorporação de novos recursos, capazes de contribuírem com a construção e formulação de propostas de intervenção que sejam centradas no indivíduo (MÂNGIA et al, 2008).

O estudo apresentou na leitura das respostas dos profissionais participantes da pesquisa um déficit no que tange a compreensão das políticas públicas de saúde. Ressalta-se também a necessidade de efetivação destas nos serviços de saúde. É necessário reforçar a inserção do usuário no serviço de saúde ao participar de forma ativa do seu processo de cuidado e busca por execução de seus direitos.

\section{REFERÊNCIAS}

BARRA, DCC; LANZONI, GMM; MALISKA, ICA; SEBOLD, LF; MEIRELLES, BHS.

Processo de viver humano e a enfermagem sob a perspectiva da vulnerabilidade. Acta Paul Enferm, 2010.

BERTOLOZZI, MR; NICHIATA, LYI; TAKAHASHI, RF; CIOSAK, SI; HINO, P; VAL, LF; GUANILLO, MCLTU; PEREIRA, EG. Os conceitos de vulnerabilidade e adesão na Saúde Coletiva. Rev Esc Enferm. São Paulo, 2009. 


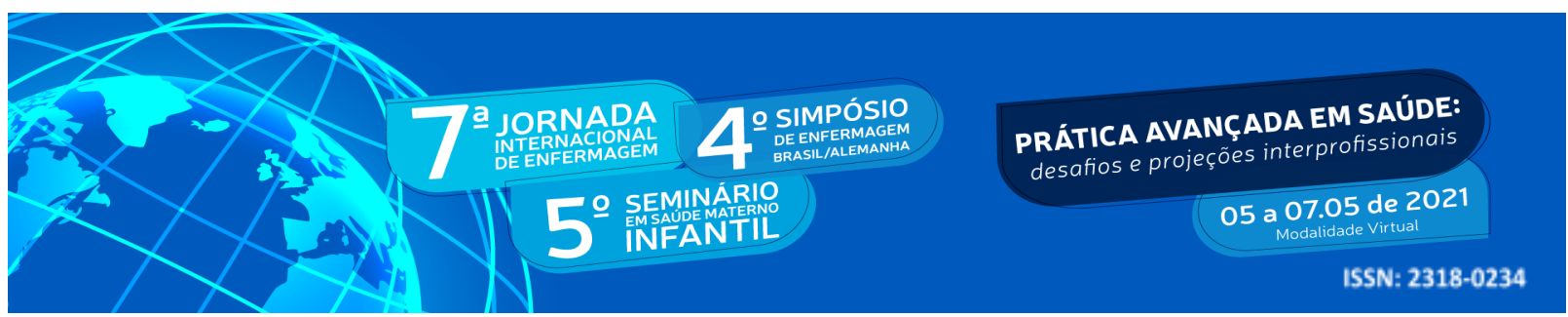

BRASIL, Senado Federal. Decreto nº 7.508 de 28 de junho de 2011. Brasília: Senado Federal, 2011.

BRASIL. Ministério da Saúde. 8080/90. Lei Orgânica da Saúde. Brasília, 1990.

CARMO, M.E; GUIZARDI, F.L. O conceito de vulnerabilidade e seus sentidos para as políticas públicas de saúde e assistência social. Cad. Saúde Pública 2018; 34(3):e00101417.

KLASSMANN, Nathan Ortiz; CARBONAI, Davide. O saneamento básico no Rio Grande do Sul sob a perspectiva do ranking nacional do saneamento. Gestão pública: casos, análises e práticas. Porto Alegre: Pubblicato, 2020. p. 185-193, 2020.

MÂNGIA, E. F.; MURAMOTO, M. T. Itinerários terapêuticos e construção de projetos terapêuticos cuidadores. Rev. Ter. Ocup. Univ. São Paulo, v. 19, n. 3, p. 176-182, set./dez. 2008 .

MINAYO, MCS. Desafio do conhecimento. $5^{\text {a }}$ ed. São Paulo (SP): Hucitec-Abrasco; 2010.

NICHIATA, LYI; BERTOLOZZI, MR; TAKAHASHI, RF; FRACOLLI, LA. A utilização do conceito "vulnerabilidade" pela Enfermagem. Rev Latino Enfermagem. Setembro-outubro de 2008 .

SCHRAIBER, Lilia Blima. Necessidades de saúde, políticas públicas e gênero: a perspectiva das práticas profissionais. Ciência \& Saúde Coletiva, v. 17, p. 2635-2644, 2012.

SILVA, K.; BEZERRA, A.F.B; TANAKA, O.Y. Direito à saúde e integralidade: uma discussão sobre os desafios e caminhos para sua efetivação. Comunicação saúde educação. v.16, n.40, p.249-59, jan./mar. 2012. 


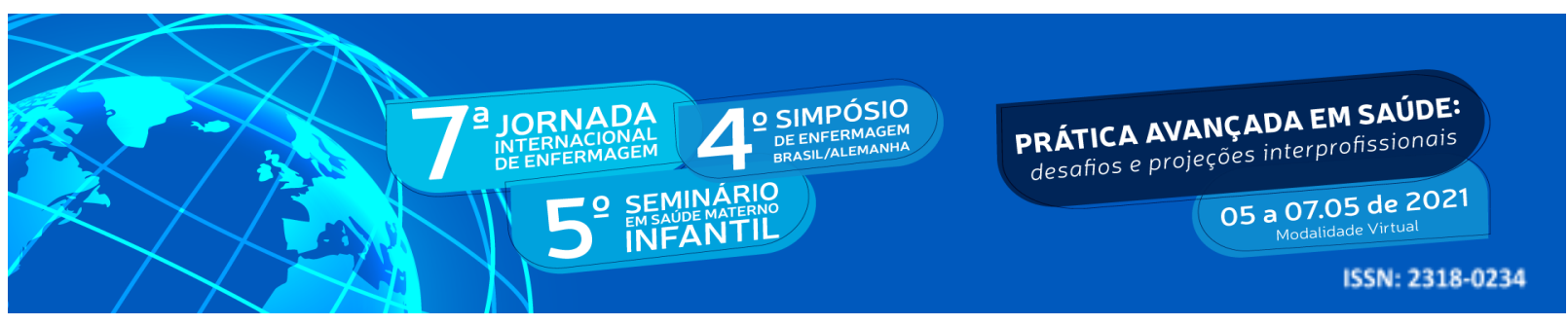

VASCONCELOS, MFF; FELIX, J; GATTO, GMS. Saúde da mulher: o que é poderia ser diferente? Rev. psicol. polít. [online]. 2017, vol.17, n.39, pp. 327-339. 